\title{
Palliative care needs in COPD patients with or without cancer: an epidemiological study
}

\author{
Cornelia Meffert ${ }^{1}$, Isaak Hatami ${ }^{2}$, Carola Xander $^{1}$ and Gerhild Becker ${ }^{1}$
}

\author{
Affiliations: \\ ${ }^{1}$ Department of Palliative Care, University Medical Centre Freiburg, Freiburg, Germany. \\ ${ }^{2}$ Department of Anaesthesiology, Intensive Care and Pain Therapy, St. Vincent Hospital, Landau, Germany.

\section{Correspondence:} \\ Gerhild Becker, University Medical Centre Freiburg, Department of Palliative Care, Palliative Care Research \\ Group, Robert-Koch-Str. 3, 79106 Freiburg, Germany. \\ E-mail: gerhild.beckerवuniklinik-freiburg.de
}

ABSTRACT Chronic obstructive pulmonary disease (COPD) is a growing cause of morbidity and mortality worldwide. However, many patients with severe COPD do not receive adequate palliative care. The main goals of our study were to identify the percentage of hospital patients with palliative care needs, particularly those who suffer from COPD.

Data were collected prospectively from inpatients at the University Medical Centre Freiburg (Freiburg, Germany). Based on the World Health Organization definition of palliative care, the treating physician reported for each patient discharged whether the patient had palliative care needs or not. Data from 39849 patients could be analysed, of which 1455 were suffering from COPD.

Of all COPD patients, $9.1 \%$ had palliative care needs. In COPD patients with palliative care needs, hospital stay was significantly longer (13.7 versus 10.3 days) than in the group without palliative care needs, and significantly more patients died during their hospital stay ( $8.3 \%$ versus $3.7 \%$ ). The presence of metastases was the highest risk factor for developing palliative care needs (OR 4.18). Furthermore, a main diagnosis of COPD implied an increased probability of palliative care needs (OR 1.87).

Our results show that COPD patients have a high risk of developing palliative care needs. Further efforts are required to provide palliative care to COPD patients.

@ERSpublications

Patients with COPD as a main diagnosis or a comorbid condition have a high risk of developing palliative care needs http://ow.ly/LJk1Y

Editorial comment in: Eur Respir J 2015; 46: 596-598 [DOI: 10.1183/13993003.00987-2015]

Received: Nov 102014 | Accepted after revision: April 03 2015 | First published online: May 282015

Conflict of interest: None declared.

Support statement: This research was supported by Roche Pharma (Grenzach-Wyhlen, Germany).

Copyright @ERS 2015 


\section{Introduction}

Chronic obstructive pulmonary disease (COPD) is an important and growing cause of morbidity worldwide and was the fifth leading cause of mortality in 2010 [1]. Studies in USA have reported a doubled death rate from COPD over the past two decades $[2,3]$. Particularly in critically ill patients, the presence of COPD is a high risk factor for increased mortality [4].

COPD is a chronic and progressive disease which is often aggravated by episodes of increased respiratory symptoms. Those exacerbations produce a significant and permanent impairment of health status [5]. Patients with advanced COPD not only suffer from multiple physically burdensome symptoms (e.g. dyspnoea and fatigue), but also from high levels of depression and anxiety [6]. The treatment of end-stage COPD continues to focus on life-prolonging treatment options (e.g. mechanical ventilation). Often, this maximum-therapy approach only provides modest relief of disabling physical symptoms. The emotional and psychosocial suffering of patients and their families often remains unnoticed and is not adequately relieved. Despite the high morbidity and mortality associated with severe COPD, many patients do not receive adequate palliative care [7].

The primary goal of palliative care is to ensure quality of life for patients with incurable, life-limiting diseases and their caregivers. This is true for all patients, regardless of their disease. Core elements of palliative care are: 1) to provide relief from pain and other distressing symptoms; 2) to integrate the psychological and spiritual aspects of patient care; 3) to offer a support system to help patients live as actively as possible until death; and 4) to offer a support system to help the family cope during the patient's illness and in their own bereavement [8].

Nowadays, in most institutions, patients with incurable cancer receive interventions for symptom control and pain management. However, patients with advanced COPD often do not receive palliative care until late in the course of treatment [9]. In a cross-sectional observational study, JANsSEN et al. [10] found that only $1.9 \%$ of patients with advanced COPD were treated with strong opioids such as morphine and $57.1 \%$ reported severe dyspnoea. Compared to patients with lung cancer, COPD patients receive fewer opiates and benzodiazepine [11] and more frequently die in intensive care units, on mechanical ventilation and with dyspnoea [7].

Ideally, palliative care should be implemented as soon as an incurable disease is diagnosed, not only in the imminent terminal phase [8]. Nevertheless, the early identification of palliative care needs (PCNs) in hospital-treated COPD patients remains an insufficiently investigated topic of research. Therefore, the main goals of our study were to identify the percentage of hospital patients with PCNs and particularly those who suffer from COPD.

\section{Material and methods}

\section{Patients and data sampling}

Data were collected prospectively from patients at the University Medical Centre Freiburg (UMCF) (Freiburg, Germany), one of the largest hospitals in Europe, with 1479 hospital beds in 42 clinical departments. Sampling took place from January 2004 to May 2005. At the time of the study, the hospital had no specialised palliative care service for in-house consultations and no palliative care unit.

For each patient discharged or transferred from a UMCF ward, electronic data sampling by the attending physician was mandatory. For the purposes of our study, we modified the file mask of the electronic discharge management by adding the dichotomous question: "Does or did this patient have palliative care needs?" For each patient, this question had to be answered by the treating physician at discharge. When the physician clicked on the question, a text box appeared explaining how PCN was defined, using the World Health Organization (WHO) definition: "Palliative care is the active total care of patients whose disease is not responsive to curative treatment. Control of pain, of other symptoms, and of psychological, social, and spiritual problems is paramount" [12]. Some aspects of PCNs (e.g. pain, fatigue, dyspnoea, nausea/vomiting, constipation, anxiety and depression) were also specified in the text box. Without answering the question about PCN, the electronic discharge management could not be completed, thereby guaranteeing a nearly $100 \%$ response rate. In addition, a special briefing was arranged for all physicians responsible for discharge management. In these mandatory instruction sessions (conducted before the observation period), the purpose of the study was illustrated and PCNs were explained in detail. The 1990 WHO definition of palliative medicine was introduced to the physicians and they were trained to correctly classify patients. These instructional sessions lasted $\sim 1.5 \mathrm{~h}$. They were offered repeatedly in the first 4 weeks of the 3-month pilot period to ensure that every physician responsible for discharge management was given the necessary briefing. If the treatment team changed during the study period, the new team member was briefed as well.

During the study period, data were collected from 100679 records. Data collection included patients who were transferred to another ward or another department within the hospital. To prevent a bias toward 
patients who had longer hospital stays and were transferred to different wards several times, only the data from the last discharge when leaving the hospital were included. This limited data to 39849 cases. 1455 of these patients were suffering from COPD. COPD was clinically diagnosed at discharge from the hospital; electronic discharge data did not include functional data concerning the severity of COPD.

The study was piloted from September 15, 2003, to December 31, 2003. In the pilot period, the quality of classification (PCN: yes/no) was repeatedly controlled by two members of the research team by comparing classifications with the medical records. In cases of inconsistencies, each patient was discussed with the physician responsible for the classification. The study team visited all wards included in the study on a weekly basis to answer questions or discuss problems.

To test the validity of the data, different quality control processes were performed after data collection. Source data verification, including checking medical records, was performed for 500 randomly selected patients (250 patients with PCNs and 250 without PCNs) by two independent reviewers, which showed that in $90 \%$ of patients with PCNs and in almost $100 \%$ of patients without PCNs, classification by the physicians responsible for dismissal was plausible.

More details of the study design are described elsewhere [13].

\section{Study questions and statistical analyses}

The main outcome measure of the study was the identification of the percentage of patients with PCNs in a large acute care hospital. Secondary outcome measures included the proportion of COPD patients with PCNs along with the measures described below.

Data were analysed with SPSS software (SPSS for Windows, version 21.0; IBM, Armonk, NY, USA). The t-test was used for interval-scaled variables; associations between dichotomous variables were tested by the Chi-squared statistic. In addition, a binary logistic regression analysis (method: forward stepwise) was performed in order to identify the probability of COPD patients having PCNs, according to physicians' assessments. All tests were two-tailed, and $\mathrm{p}<0.05$ was considered statistically significant.

\section{Ethics}

The study protocol was approved by the ethics committee of Freiburg University Hospital and the data security official. Regulations of the European Data Protection Directive [14] were followed. The study was conducted according to the Declaration of Helsinki [15].

\section{Results}

Altogether 1455 (3.7\%) of all patients admitted to the UMCF had COPD. Of the 1455 COPD patients, 267 patients $(18.4 \%)$ were discharged from the hospital with COPD as a main diagnosis. The other 1188 patients $(81.6 \%$ of all COPD patients) had COPD as a comorbid condition but not as a principal diagnosis.

During the study period, 9.1\% of all COPD patients (132 out of 1455) had PCNs. 104 (78.8\%) of these palliative care patients were treated in the department of internal medicine (which includes respiratory and cardiac care units), $14(10.6 \%)$ in the department of radiotherapy, nine $(6.8 \%)$ in the department of surgery, three $(2.3 \%)$ in the department of neurology and neurosurgery and two $(1.5 \%)$ in the department of gynaecology. Patient characteristics, such as the presence of PCNs, number of days in the hospital, discharge status, and palliative treatment as a part of their therapy are listed in table 1.

Only three $(2.3 \%)$ out of 132 COPD patients with PCNs received palliative treatment. In the group with PCNs, patients' hospital stay was significantly longer (13.7 days versus 10.3 days; $\mathrm{p}=0.001$ ) and significantly more patients died during their hospital stay ( $8.3 \%$ versus $3.7 \%$; $=0.011$ ) (table 1$)$.

Table 2 provides a summary of the accompanying diseases in COPD patients. Data show that the majority of patients with PCNs had cancer $(51.5 \%$ of all COPD patients with PCNs), followed by those with metastases $(33.3 \%)$ and coronary heart disease $(25.0 \%)$. The proportion of patients with PCNs relative to all patients of a diagnostic group was highest in patients with metastases (20.5\%) and patients who suffer from dementia (20.0\%) and/or depression (21.9\%).

\section{Subgroup: patients with COPD as main diagnosis}

Out of the 267 patients with COPD as a principal diagnosis, 31 (11.6\%) had PCNs. Patient characteristics of this subgroup are shown in table 3 . Comorbidities are listed in table 4. 
TABLE 1 Patient characteristics, palliative treatment, hospital stay and discharge status

\begin{tabular}{lccc} 
& $\begin{array}{c}\text { COPD patients with } \\
\text { PCNs }\end{array}$ & $\begin{array}{c}\text { COPD patients } \\
\text { without PCNs }\end{array}$ & p-value \\
\hline Subjects & 132 & 1323 & \\
Age in years & $68.8 \pm 10.6$ & $67.2 \pm 10.7$ & 0.113 \\
Male & $94(71.2)$ & $942(71.2)$ & 0.998 \\
Presence of relatives & $70(53.0)$ & $810(61.2)$ & 0.066 \\
Palliative treatment & $3(2.3)$ & $3(0.2)$ & $<0.001$ \\
Length of hospital stay days & $13.7 \pm 11.7$ & $10.3 \pm 10.9$ & 0.001 \\
Deceased & $11(8.3)$ & $49(3.7)$ & 0.011 \\
Discharged home & $92(69.7)$ & $1079(81.6)$ & 0.001 \\
Discharged to another acute care hospital or & $23(17.4)$ & $168(12.7)$ & 0.125 \\
rehabilitation centre & & & \\
Discharged elsewhere le.g. nursing home) & $6(4.5)$ & $27(2.0)$ & 0.065 \\
\hline
\end{tabular}

Data are presented as $\mathrm{n}$, mean \pm SD or $\mathrm{n}(\%)$, unless otherwise stated. COPD: chronic obstructive pulmonary disease; PCNs: palliative care needs.

\section{Logistic regression analysis}

To identify the probability of COPD patients having PCNs, we performed a binary logistic regression analysis based on the entire sample of COPD patients $(n=1455)$. In the regression model, we included sex, age $\geqslant 65$ years, COPD as a main diagnosis, diagnosis of other incurable illnesses (such as cancer, chronic heart failure and liver cirrhosis), depression and presence/absence of relatives.

By these means, we discovered that the presence of metastases was the highest risk factor for developing PCNs (OR 4.18, 95\% CI 2.73-6.40). Furthermore, a main diagnosis of COPD implied an increased probability of PCNs (OR 1.87, 95\% CI 1.18-2.96). Thus, patients with metastatic cancer and COPD as a principal diagnosis had an increased probability of being identified as having PCNs (OR 7.82). For COPD patients who also suffered from depression, the odds ratio of developing PCNs was 2.56 (95\% CI 1.066.18) and the absence of relatives increased this probability by an odds ratio of 0.64 (95\% CI $0.44-0.93$ ).

\section{Discussion}

In order to be able to offer need-based early palliative care, patients' PCNs must be identified at an early stage. One of the main goals of our study was to identify the percentage of COPD patients with PCNs in a large acute-care hospital. Our results show that COPD patients, either with COPD as a main diagnosis or with comorbid COPD had a high risk of developing PCNs.

TABLE 2 Comorbidities in chronic obstructive pulmonary disease patients with and without palliative care needs (PCNs) and frequency of PCNs in these diagnostic groups

$\begin{array}{cccc}\begin{array}{c}\text { Patients } \\ \text { with PCNs }\end{array} & \begin{array}{c}\text { Patients } \\ \text { without PCNs }\end{array} & \text { p-value } & \begin{array}{c}\text { Proportion of patients with } \\ \text { PCNs relative to all patients } \\ \text { of the diagnostic group } \%\end{array}\end{array}$

\begin{tabular}{lcccc}
\hline Subjects & 132 & 1323 & & \\
Malignant neoplasm & $68(51.5)$ & $470(35.5)$ & $<0.001$ & 12.6 \\
Metastases & $44(33.3)$ & $171(12.9)$ & $<0.001$ & 20.5 \\
Bronchial carcinoma & $29(22.0)$ & $245(18.5)$ & 0.334 & 10.6 \\
Coronary heart disease & $33(25.0)$ & $347(26.2)$ & 0.759 & 8.7 \\
Chronic heart failure & $18(13.6)$ & $142(10.7)$ & 0.309 & 71.3 \\
Diabetes mellitus & $15(11.4)$ & $175(13.2)$ & 0.544 & 6.9 \\
Chronic renal failure & $11(8.3)$ & $156(11.8)$ & 0.235 & 17.0 \\
Acute renal failure & $8(6.1)$ & $39(2.9)$ & 0.054 & 14.7 \\
Liver cirrhosis & $5(3.8)$ & $29(2.2)$ & 0.247 & 21.9 \\
Depression & $7(5.3)$ & $25(1.9)$ & 0.011 & 20.0 \\
Dementia & $4(3.0)$ & $16(1.2)$ & 0.087 &
\end{tabular}

Data are presented as $\mathrm{n}$ or $\mathrm{n}(\%)$, unless otherwise stated. Note that the percentages in the first two columns do not equal $100 \%$, as diagnostic groups are not disjunctive. 
TABLE 3 Chronic obstructive pulmonary disease as main diagnosis: patient characteristics, palliative treatment, hospital stay and discharge status

\begin{tabular}{lccc} 
& Patients with PCNs & Patients without PCNs & p-value \\
\hline Subjects & 31 & 236 & \\
Age years & $70.1 \pm 9.4$ & $67.6 \pm 9.9$ & 0.191 \\
Male & $19(61.3)$ & $144(61.0)$ & 0.977 \\
Presence of relatives & $11(35.5)$ & $123(52.1)$ & 0.082 \\
Palliative treatment & $1(3.2)$ & $0(0.0)$ & 0.006 \\
Length of hospital stay days & $14.5 \pm 9.9$ & $10.4 \pm 11.4$ & 0.056 \\
Deceased & $1(3.2)$ & $6(2.5)$ & 0.823 \\
Discharged home & $24(77.4)$ & $211(89.4)$ & 0.053 \\
$\quad$ Discharged to another acute care hospital & $6(19.4)$ & $12(5.1)$ & 0.003 \\
$\quad$ or rehabilitation centre & & & \\
Discharged elsewhere & $0(0.0)$ & $7(3.0)$ & 0.331
\end{tabular}

Data are presented as $n$, mean \pm SD or $n(\%)$, unless otherwise stated. PCNs: palliative care needs.

PCNs were defined using the widely accepted WHO definition of palliative care [12]. We did not use the "surprise" question ("Would you be surprised if this patient died in the next year?") to identify patients' PCNs. This question suggests that patients are nearing the end of life. However, COPD patients may experience high symptom distress for much longer than 12 months. BAUSEWEIN et al. [16] compared the symptom burden in patients with advanced lung cancer and advanced COPD. They found similar PCNs in both groups, but a median survival of 107 days in patients with cancer and 589 days in patients with COPD [16]. Thus, the "surprise" question would not have been effective in identifying PCNs in COPD patients.

Despite advances in care, the COPD epidemic persists, causing $>120000$ deaths per year in USA alone. Population-based surveys show that as many as 24 million people in USA have airflow limitation consistent with COPD [17]. Despite the availability of effective treatments for COPD, no existing therapy halts or reverses the progressive decline in lung function that is characteristic of this condition. COPD is an illness with no cure. Due to the irreversible nature of COPD, the aim of treatment is to reduce symptoms, increase functioning, and improve patients' quality of life [18]. However, evidence suggests that existing healthcare provision for COPD patients is generally reactive and focuses on acute exacerbations [19]. Patients with end-stage COPD commonly have unmet needs, such as dealing with a high daily symptom burden and emotional distress [20]. They have a significantly impaired quality of life and emotional wellbeing, and often they do not receive holistic care appropriate to their needs [21]. A comparison with cancer patients shows a lack of palliative care provision for patients with advanced-stage COPD despite the latter having poor prognosis, intolerable dyspnoea, greater disability, poor quality of life and higher levels of depression [22].

TABLE 4 Chronic obstructive pulmonary disease (COPD) as main diagnosis: comorbidities in COPD patients with and without palliative care needs (PCNs) and frequency of PCNs

$\begin{array}{cccc}\begin{array}{c}\text { Patients } \\ \text { with PCNs }\end{array} & \begin{array}{c}\text { Patients } \\ \text { without PCNs }\end{array} & \text { p-value } & \begin{array}{c}\text { Proportion of patients with } \\ \text { PCNs relative to all patients } \\ \text { of the diagnostic group } \%\end{array}\end{array}$

\begin{tabular}{llccc}
\hline Subjects & 31 & 236 & & \\
Malignant neoplasm & $5(16.1)$ & $22(9.3)$ & 0.237 & 18.5 \\
Metastases & $2(6.5)$ & $2(0.8)$ & 0.016 & 50.0 \\
Coronary heart disease & $7(22.6)$ & $44(18.6)$ & 0.600 & 13.7 \\
Chronic heart failure & $7(22.6)$ & $21(8.9)$ & 0.019 & 25.0 \\
Diabetes mellitus & $3(9.7)$ & $32(13.6)$ & 0.547 & 8.6 \\
Chronic renal failure & $5(16.1)$ & $27(11.4)$ & 0.450 & 15.6 \\
Acute renal failure & $1(3.2)$ & $6(2.5)$ & 0.823 & 14.3 \\
Depression & $3(9.7)$ & $7(3.0)$ & 0.064 & 30.0 \\
Dementia & $1(3.2)$ & $2(0.8)$ & 0.238 & 33.3
\end{tabular}

Data are presented as $\mathrm{n}$ or $\mathrm{n}(\%)$, unless otherwise stated. Note that the percentages in the first two columns do not equal $100 \%$, as diagnostic groups are not disjunctive. 
In a cross-sectional, general population-based survey of 11985 subjects from 17 countries, JANSON et al. [23] found that severe COPD has a greater negative impact on physical and mental health status than self-reported cardiovascular disease and diabetes. COPD patients in their last year of life are likely to suffer from chronic dyspnoea (98\%), fatigue (96\%), low mood (77\%) and pain (70\%). Of these symptoms, dyspnoea is partly relieved in $50 \%$ of patients; however, low mood is relieved in only $8 \%$ of all cases and is not treated in $82 \%$ [24].

In our study analysing 39849 hospital stays over a period of 17 months, $9.1 \%$ of all COPD patients (132 out of 1455) were classified as having PCNs. Logistic regression analysis showed that a main diagnosis of COPD increases the statistical probability of having PCNs by a factor of nearly two. The most frequent comorbidities of those patients were coronary heart disease (22.6\%) and chronic heart failure (22.6\%). These findings confirm the results of other epidemiological studies which have shown that COPD is frequently associated with comorbidities, the most serious and prevalent being cardiovascular disease and lung cancer $[25,26]$. Comorbidities contribute to patients' health status, and the presence of one disease significantly affects the prognosis of the other [27].

In our study, the majority of COPD patients with PCNs had cancer (51.5\%), followed by those with metastases (33.3\%). However, in the group of COPD patients without PCNs, 35.5\% had a malignant tumour and $12.9 \%$ a metastatic disease. At first glance, this may appear to be contradictory. Yet this discrepancy suggests that physicians may have decided to attribute PCNs based on the severity of patients' symptoms or quality of life rather than on general characterisations of the disease (i.e. malignant versus benign).

Nevertheless, the fact that most COPD patients had cancer, some even with metastases, is a confounding factor which cannot be ignored. Conversely, research literature provides ample evidence that lung cancer and COPD are a common combination [28] and that the suffering of patients with COPD is comparable to that of patients with cancer [29]. Patients with advanced COPD experience similar deterioration in symptoms and quality of life as those with advanced malignancy [30]. For this reason, we do not see any reason why PCNs should be considered mainly within a disease-specific group. We are faced with an ageing population and a growing number of people who are dying with multiple comorbid conditions, such as cancer and COPD [31].

Analysing the frequency of PCNs within different comorbidities, PCNs were highest in COPD patients with metastases, dementia and depression. Emotional disturbances such as depression or anxiety are common among patients with COPD [32], and an excess of depressive symptoms is associated with increased mortality risk [33]. VAN MANEN et al. [34] found a strong relationship between increased depression scores and factors such as living alone. In our study, the absence of relatives and depression were shown to be clear risk factors for developing PCNs, which underlines the importance of psychosocial issues in the palliative care of COPD patients.

For more than 15 years, experts have been promoting the idea that palliative care should be provided on the basis of need rather than diagnosis [35]. In light of such demands, the findings of our study confront us with rather alarming figures: only $2.3 \%$ of COPD patients with PCNs actually received palliative care; in $97.7 \%$ these needs remained unmet.

A recent official statement of the American Thoracic Society on palliative care for patients with respiratory diseases strongly endorsed the concept that palliative care should be available to patients at all stages of their illness and should be individualised based on the needs and preferences of the patient and the patient's family [36]. They suggested early palliative care, together with curative and restorative care, when there is an increased intensity of symptoms such as breathlessness, pain, fatigue and depression [37].

For patients with nonsmall cell lung cancer, TEmel et al. [38] showed that early palliative care improved patients' quality of life, reduced the incidence of depression and decreased the number of aggressive (and generally futile) therapies at the end of life. In addition, early palliative care increased patients' survival time [38]. In a randomised study of patients suffering from different cancer entities, ZiMMERMANN et al. [39] found that patients who received early palliative care not only had a better quality of life but also a higher level of satisfaction with their treatment compared to those in the control group, who were not offered early palliative care.

Undoubtedly, an increasing number of cancer patients, and above all lung cancer patients, receive palliative care alongside standard oncology care. This has become widely accepted. However, it cannot be denied that COPD patients are also in need of palliative care. Patients dying of COPD may experience physical symptoms and psychosocial distress at least as severe as patients with lung cancer [40]. Further efforts are required to integrate palliative care into the treatment of COPD patients in acute-care hospitals. We hope that our study contributes to encourage physicians and caregivers to recognise the PCNs of COPD patients and to meet them not only during the latest stage of the disease. 


\section{Limitations}

Our study had a number of inherent limitations. The absence of lung function data and detailed information about the functional capacities of the patients did not allow for discrimination between different degrees of severity of COPD.

A large part of our study population had a diagnosis of cancer and metastasis. We cannot estimate whether there may be a synergistic effect on health status and PCNs for some disease combinations, such as cancer and COPD. However, a recently published study found that effects of interaction between comorbidities on several health outcomes were primarily additive, not synergistic [41]. Additional research is needed to assess to what extent COPD on the one hand and cancer on the other hand contribute to the development of PCNs in COPD patients.

We asked the treating physicians about patients' PCNs and not the patients themselves or their relatives. As we conducted an epidemiological study with close to 40000 patients, the latter was not possible. On the one hand, this might be a source of bias. On the other hand, it is well known that there is poor communication between clinicians and patients about the life-limiting nature of COPD and the terminal prognosis in advanced stages of the disease [42]. As "palliative care needs" are associated with mortality and death, we assume that patients would most probably deny them.

Although the analysis is based on data from nearly 40000 patients, our study is only a single-centre analysis. When completing the discharge form, physicians had to report whether the patient had PCNs or not. The accuracy of physicians' assessments was a key issue, and therefore all physicians involved in the study were specially trained and continuously supervised. This would not have been possible in a multicentre setting.

In the database, patients with multiple stays in the hospital were analysed as separate cases. This may have been a source of bias. Conversely, analysing multiple stays of the same person as only one case could also present a source of bias. Which stay should be regarded as the relevant one? In order to more accurately calculate the number of beds needed for patients with PCNs, we decided to analyse multiple stays as independent.

A definition of PCNs is complex because palliative care can be divided into two components. First, there is general palliative care that can be provided by generalists in their daily work. Second, there are specialist palliative care physicians and nurses who are trained specifically to handle complex palliative situations. To keep the survey clear and simple, we intentionally used the widely accepted WHO definition of palliative care [12] without differentiating between the primary and secondary levels of palliative care. To what extent generalists may satisfy basic needs for palliative care and how much specialist palliative care is needed for complex situations cannot be answered by our data.

\section{Conclusions}

Given the ageing population and associated increase in the number of patients requiring palliative care, it is crucial that PCNs are detected in early stages of cancerous and noncancerous life-threatening diseases. In general, such patients are likely to suffer from pain, dyspnoea and other burdensome physical symptoms and require psychosocial or spiritual support. They are highly vulnerable, both psychologically and physically, and they are in need of palliative care. Our results show that COPD patients, either with COPD as a main diagnosis or as a comorbid condition have a high risk of developing PCNs. Furthermore, the absence of relatives and the presence of psychological ailments such as depression were shown to be clear risk factors, which underlines the importance of psychosocial issues in palliative care. As there are comparatively few data on PCNs in noncancer conditions such as COPD, further research is needed.

\section{Acknowledgement}

The authors would like to thank Karin Jors (Department of Palliative Care, University Medical Centre Freiburg, Germany) for her support in revising the manuscript as a native English speaker.

\section{References}

Murray CJ, Lopez AD. Measuring the global burden of disease. N Engl J Med 2013; 369: 448-457.

2 Jemal AD, Ward E, Hao Y, et al. Trends in the leading causes of death in the United States, 1970-2002. JAMA 2005; 294: 1255-1259.

3 Holguin F, Folch E, Redd SC, et al. Comorbidity and mortality in COPD-related hospitalizations in the United States, 1979 to 2001. Chest 2005; 128: 2005-2011.

4 Funk G-C, Bauer P, Burghuber OC, et al. Prevalence and prognosis of COPD in critically ill patients between 1998 and 2008. Eur Respir J 2013; 41: 792-799.

5 Miravitlles M, Calle M, Alvarez-Gutierrez F, et al. Exacerbations, hospital admissions and impaired health status in chronic obstructive pulmonary disease. Qual Life Res 2006; 15: 471-480.

6 Goodridge DM, Marciniuk DD, Brooks D, et al. End-of-life care for persons with advanced chronic obstructive pulmonary disease: report of a national interdisciplinary consensus meeting. Can Respir J 2009; 16: e51-e53. 
Curtis JR. Palliative and end-of-life care for patients with severe COPD. Eur Respir J 2008; 32: 796-803.

8 World Health Organization. WHO Definition of Palliative Care. www.who.int/cancer/palliative/definition/en/ Date last accessed: February 15, 2013.

9 Sorenson HM. Palliative care for lung disease: start early, stay late. Lancet Respir Med 2013; 1: 279-280.

10 Janssen DJA, Spruit MA, Uszko-Lencer NH, et al. Symptoms, comorbidities, and health care in advanced chronic obstructive pulmonary disease or chronic heart failure. J Palliat Med 2011; 14: 735-743.

11 Yohannes AM. Disability in patients with COPD. Chest 2014; 145: 200-202.

12 World Health Organization. Cancer Pain Relief and Palliative Care. Technical Report Series 804. Geneva, World Health Organization, 1999.

13 Becker G, Hatami I, Xander C, et al. Palliative cancer care: an epidemiologic study. J Clin Oncol 2011; 29: $646-650$.

14 Directive 95/46/EC of the European Parliament and of the Council of 24 October 1995 on the protection of individuals with regard to the processing of personal data and on the free movement of such data. www.cdt.org/ privacy/eudirective/EU_Directive_html Date last accessed: January 8, 2014.

15 World Medical Association. Declaration of Helsinki. www.wma.net/en/20activities/10ethics/10helsinki/index.html Date last accessed: January 8, 2014.

16 Bausewein C, Booth S, Gysels M, et al. Understanding breathlessness: cross-sectional comparison of symptom burden and palliative care needs in chronic obstructive pulmonary disease and cancer. J Palliat Med 2010; 13: 1109-1118.

17 Punturieri A, Croxton TL, Weinmann GG, et al. Chronic obstructive pulmonary disease: a view from the NHLBI. Am J Respir Crit Care Med 2008; 178: 441-443.

18 Vögele C, von Leupoldt A. Mental disorders in chronic obstructive pulmonary disease (COPD). Respir Med 2008; 102: 764-773.

19 Elkington H, White P, Addington-Hall J, et al. The last year of life of COPD: a qualitative study of symptoms and services. Respir Med 2004; 98: 439-445.

20 Janssen DJA, McCormick JR. Palliative care and pulmonary rehabilitation. Clin Chest Med 2014; 35: 411-421.

21 Gore JM, Brophy CJ, Greenstone MA. How well do we care for patients with end stage chronic obstructive pulmonary disease (COPD)? A comparison of palliative care and quality of life in COPD and lung cancer. Thorax 2000; 55: 1000-1006.

22 Yohannes AM. Palliative care provision for patients with chronic obstructive pulmonary disease. Health Qual Life Outcomes 2007; 5: 17.

23 Janson C, Marks G, Buist S, et al. The impact of COPD on health status: findings from the BOLD study. Eur Respir J 2013; 42: 1472-1483.

24 Dean MM. End-of-life care for COPD patients. Prim Care Respir J 2008; 17: 46-50.

25 Piquet J, Chavaillon J-M, David P, et al. High-risk patients following hospitalisation for an acute exacerbation of COPD. Eur Respir J 2013; 42: 946-955.

26 Decramer M, Janssens W. Chronic obstructive pulmonary disease and comorbidities. Lancet Respir Med 2013; 1: $73-83$.

27 Vestbo J, Anderson J, Brook RD, et al. The Study to Understand Mortality and Morbidity in COPD (SUMMIT) study protocol. Eur Respir J 2013; 41: 1017-1022.

28 Abal Arca J, Parente Lamelas I, Almazán Ortega R, et al. Cáncer de pulmón y EPOC: una asociación frecuente [Lung cancer and COPD: a common combination]. Arch Bronconeumol 2009; 45: 502-507.

29 Weingaertner V, Scheve C, Gerdes V, et al. Breathlessness, functional status, distress, and palliative care needs over time in patients with advanced chronic obstructive pulmonary disease or lung cancer: a cohort study. $J$ Pain Symptom Manage 2014; 48: 569-581 e1.

30 Rocker G, Simpson AC, Horton R. Palliative care in advanced lung disease: the challenge of integrating palliation into everyday care. Chest 2015; (in press [DOI: 10.1378/chest.14-2593]).

31 Murtagh FE, Vinen K, Farrington K, et al. Living and dying with COPD. End of life trajectories across conditions. BMJ 2011; 342: d989.

32 Willgoss TG, Yohannes AM. Anxiety disorders in patients with COPD: a systematic review. Respir Care 2013; 58: 858-866.

33 Ng T, Niti M, Tan W, et al. Depressive symptoms and chronic obstructive pulmonary disease: effect on mortality, hospital readmission, symptom burden, functional status, and quality of life. Arch Intern Med 2007; 167: 60-67.

34 Van Manen JG, Bindels PJE, Dekker FW, et al. Risk of depression in patients with chronic obstructive pulmonary disease and its determinants. Thorax 2002; 57: 412-416.

35 Higginson IJ, Addington-Hall JM. Palliative care needs to be provided on basis of need rather than diagnosis. BMJ 1999; 318: 123.

36 Lanken PN, Terry PB, DeLisser HM, et al. An official American Thoracic Society Clinical policy statement: palliative care for patients with respiratory diseases and critical illnesses. Am J Respir Crit Care Med 2008; 177: 912-927.

37 Carlucci A, Guerrieri A, Nava S. Palliative care in COPD patients: is it only an end-of-life issue? Eur Respir Rev 2012; 21: 347-354.

38 Temel JS, Greer JA, Muzikansky A, et al. Early palliative care for patients with metastatic non-small-cell lung cancer. N Engl J Med 2010; 363: 733-742.

39 Zimmermann C, Swami N, Krzyzanowska M, et al. Early palliative care for patients with advanced cancer: a cluster-randomised controlled trial. Lancet 2014; 383: 1721-1730.

40 Crawford GB, Brooksbank MA, Brown M, et al. Unmet needs of people with end-stage chronic obstructive pulmonary disease: recommendations for change in Australia. Intern Med J 2013; 43: 183-190.

41 Tinetti ME, McAvay GJ, Chang SS, et al. Contribution of multiple chronic conditions to universal health outcomes. J Am Geriatr Soc 2011; 59: 1686-1691.

42 Gardiner C, Barnes S, Small N, et al. Reconciling informed consent and 'do no harm': ethical challenges in palliative-care research and practice in chronic obstructive pulmonary disease. Palliat Med 2010; 24: 469-472. 